\title{
Older and stronger object memories are selectively destabilized by reactivation in the presence of new information
}

\author{
Boyer D. Winters, ${ }^{1}$ Mark C. Tucci, and Melynda DaCosta-Furtado \\ Department of Psychology, University of Guelph, Guelph, Ontario N1G 2W1, Canada
}

\begin{abstract}
Reactivation can destabilize previously consolidated memories, rendering them vulnerable to disruption and necessitating a process of reconsolidation in order for them to be maintained. This process of destabilization and reconsolidation has commonly been cited as a means by which established memories can be updated or modified. However, little direct evidence exists to support this view. The present study addressed this issue by analyzing the influence of novel salient information present at the time of memory reactivation on the likelihood of the reactivated memory to become destabilized and vulnerable to disruption. Rats explored sample objects and, some time later, received systemic injections of the $N$-methyl-D-aspartic acid (NMDA) receptor antagonist MK-801 or saline prior to memory reactivation. When object memories were relatively young or weakly encoded, MK-801 significantly disrupted reconsolidation regardless of the reactivation conditions. However, increasing the amount of sample object exploration or the interval between the sample phase and reactivation abolished the effect of MK-80I on reconsolidation unless salient novel contextual information was present during memory reactivation. These results highlight the dynamic nature of memory storage and retrieval and indicate an important interaction between the age and strength of a memory, its probability of being destabilized upon reactivation, and the stimulus conditions during reactivation. The essential involvement of novel encoding in destabilizing certain memories supports the idea that the reconsolidation process enables modification of existing memories.
\end{abstract}

There is now ample evidence to suggest that long-term memory storage is a much more plastic process than was once believed. Traditional consolidation theory suggests that the post-acquisition stabilization of an initially labile memory occurs only once for a given memory (McGaugh 1966, 2000; Dudai 2004). Research on the phenomenon of memory reconsolidation, however, indicates that previously consolidated memories can become labile and sensitive to disruption upon reactivation (Misanin et al. 1968; Przybyslawski and Sara 1997; Nader et al. 2000a,b; Nader 2003; Dudai 2004). This reactivation-induced destabilization, which has been demonstrated in many species and memory systems (Sara 2000; Anokhin et al. 2002; Pedreira et al. 2002; Nader 2003; Dudai 2004; Dudai and Eisenberg 2004; Eisenberg and Dudai 2004; Torras-Garcia et al. 2005; Lee et al. 2006; Lee and Everitt 2008), necessitates a new stabilization phase similar but not identical to the original consolidation phase (Dudai 2004; Lee et al. 2004; Alberini 2005; von Hertzen and Giese 2005).

It has been widely speculated that memory reconsolidation might facilitate the incorporation of new information into existing memory traces (Lewis 1979; Nader 2003; Dudai 2004; RodriguezOrtiz et al. 2005, 2008; Morris et al. 2006; Rossato et al. 2007). It remains unclear, however, whether novel information encoding at the time of reactivation is a necessary condition for memory destabilization. Although it has been suggested that reconsolidation will only occur under new encoding conditions (RodriguezOrtiz et al. 2005, 2008; Morris et al. 2006), there are many cases where explicit novel information seems to be absent from the reactivation protocol (Nader et al. 2000a; Milekic and Alberini 2002; Eisenberg and Dudai 2004; Suzuki et al. 2004; Lee et al. 2006; Rossato et al. 2006; Lee and Everitt 2008). Recent studies have

\footnotetext{
'Corresponding author.
}

E-mail bwinters@uoguelph.ca; fax (519) 837-8629.

Article is online at http://www.learnmem.org/cgi/doi/10.1101//m.1509909. shown that memory reactivation in learning tasks where animals are performing at subasymptotic levels can render memory vulnerable to disruption by the protein synthesis inhibitor anisomycin (Morris et al. 2006; Rodriguez-Ortiz et al. 2008). These reports are highly suggestive of a role for novel encoding conditions in destabilizing the original memory trace.

Recent findings indicate that memories are less likely to become destabilized if they have been strongly encoded or if a greater amount of time has passed between acquisition and reactivation (Milekic and Alberini 2002; Eisenberg and Dudai 2004; Suzuki et al. 2004; Boccia et al. 2006; Bustos et al. 2009). The current study therefore examined the effects of explicit novel information encoding during memory reactivation on the likelihood of memories of different ages and strengths to become destabilized. We predicted that explicit novel encoding conditions at the time of reactivation would be necessary to destabilize older and stronger memory traces, and that selective reconsolidation impairment could be demonstrated under such conditions. This hypothesis was assessed using the spontaneous object recognition paradigm for rats. Because this study involved systemic drug injections, we chose to use the $N$-methyl-D-aspartic acid (NMDA) receptor antagonist MK-801 ([+]-5-methyl-10,11-dihydro-SH-dibenzo[a,d] cyclohepten-5,10-imine maleate; Sigma) rather than a protein synthesis inhibitor like anisomycin. NMDA receptor activation has been shown to be important for normal consolidation of object memories in the spontaneous object recognition task (Abe et al. 2004; Winters and Bussey 2005; Barker et al. 2006), and several studies have indicated a role for NMDA receptors in reconsolidation of different types of memory (Przybyslawski and Sara 1997; Lee et al. 2006; Brown et al. 2008; Lee and Everitt 2008). Indeed, the results of Experiment 1 in the current study confirm the involvement of NMDA receptors in object memory reconsolidation. Here, we report, for the first time, data indicating an important interaction between putative boundary conditions for 
reconsolidation (age, strength of memories) and the presence of salient novel information during memory reactivation.

Results

Experiment 1: Disruption of object recognition memory by 24-h post-sample MK-801 administration requires reactivation of the memory

Experiment 1 was conducted to demonstrate reactivation-dependent reconsolidation effects in the spontaneous object recognition task. Systemic injections of the NMDA receptor antagonist MK-801 given $\sim 24 \mathrm{~h}$ after the sample phase had no effect on object recognition unless the rats were also reexposed to the sample objects during a memory reactivation phase (Fig. 1). Mixed-factorial analyses of variance (ANOVA) on the discrimination ratio from the choice phase revealed a significant main effect of drug $\left(F_{(1,9)}=\right.$ $6.51, P<0.05)$ and a significant drug by reactivation condition interaction $\left(F_{(1,9)}=\right.$ $8.74, P<0.05)$. The main effect of reactivation condition was not significant $(F<1)$. Paired-samples $t$-tests conducted as post-hoc analyses indicated that MK-801 significantly disrupted object recognition in the reactivation condition $\left(t_{(5)}=3.22, P<0.05\right)$, but not in the no-reactivation group $(t<1)$. This reactivation-specific disruption indicates that initial consolidation is complete within $24 \mathrm{~h}$ of the sample phase and that object memories must undergo NMDA receptor-dependent reconsolidation for successful post-reactivation performance in the spontaneous object recognition memory paradigm.

There were no significant effects in any of the control measures analyzed, indicating that animals in both groups and drug conditions demonstrated similar general object exploratory behavior in the sample, reactivation, and choice phases (see Tables 1-3 for the control measure values from the sample, reactivation, and choice phases, respectively).

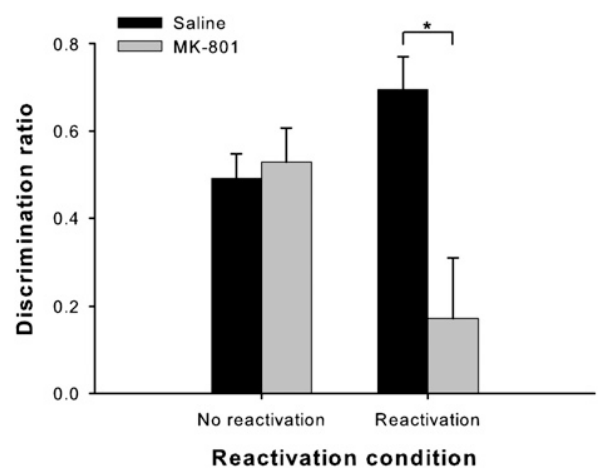

Figure 1. Object memory reconsolidation is blocked in a reactivationdependent manner by administration of the NMDA receptor antagonist MK-801 $24 \mathrm{~h}$ after the sample phase. Spontaneous object recognition performance by rats in Experiment 1 on trials in which they received systemic injections of MK-801 or saline 20 min before the reactivation phase (reactivation condition) or at the same time, but not followed by memory reactivation (no reactivation condition). MK-801 selectively disrupted memory in the reactivation condition. Data are presented as average discrimination ratio \pm SEM. $\left({ }^{*}\right) P<0.05$.

\section{Experiment 2: Salient novel information during} reactivation is not necessary to destabilize object memories following a 24-h sample-to-reactivation interval Experiment 2 was conducted to determine the effect on reconsolidation of reactivating the sample object memory trace in the presence or absence of novel contextual information. NMDA receptor antagonism disrupted reconsolidation in both reactivation conditions in Experiment 2 (Fig. 2). Mixed-factorial ANOVA on the discrimination ratio from the choice phase revealed a significant main effect of drug $\left(F_{(1,20)}=7.41, P=0.01\right)$. Neither the main effect of reactivation condition nor the interaction was significant (both $F<1$ ). Planned comparisons revealed significant differences between saline and MK-801 performance in both the standard $\left(t_{(11)}=1.94, P<0.05\right)$ and novel $\left(t_{(9)}=2.14, P<0.05\right)$ reactivation conditions. This finding, taken with the results of Experiment 1, indicates that, with a 24-h interval between sample and reactivation phases, explicit novel information encoding is not required for the object memory trace to become destabilized and sensitive to NMDA receptor blockade.

Means ( \pm SEM) for the control measures taken from the sample, reactivation, and choice phases are presented in Tables $1-3$. Of the control measures analyzed, the only significant effects were found in the reactivation phase. The reactivation phase duration analysis revealed a significant main effect of reactivation condition $\left(F_{(1,20)}=13.81, P=0.001\right)$, as rats in the novel condition took longer to reach the 10 -sec object exploration criterion (Table 2 ). This effect is presumably related to the added time spent actively exploring the context change, an indication that new information was indeed encoded in the novel reactivation condition. There were also significant main effects of drug $\left(F_{(1,20)}=7.32\right.$, $P=0.01)$ and reactivation condition $\left(F_{(1,20)}=9.37, P<0.01\right)$ in the reactivation phase total object exploration analysis. Animals in the standard condition explored slightly more than those in the novel condition (Table 2), and there was slightly higher object exploration on MK-801 trials compared with saline trials (Table 2). This latter effect is unlikely to have had much bearing on the drug effect in object recognition because the difference is a matter of 


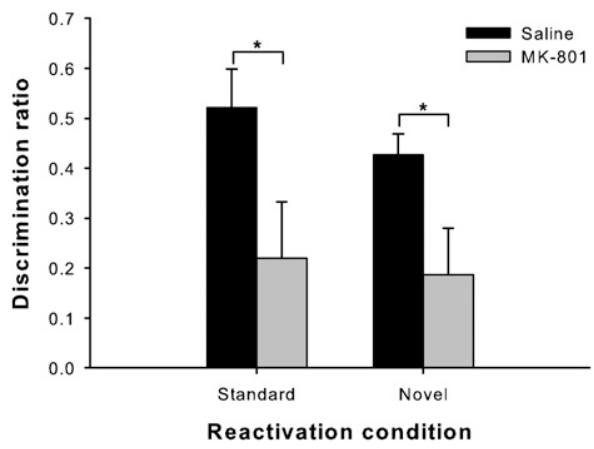

Figure 2. Relatively weak and young object memory traces are destabilized upon reactivation $24 \mathrm{~h}$ after acquisition regardless of the reactivation phase conditions. Spontaneous object-recognition performance by rats in Experiment 2 on trials in which they received systemic injections of the NMDA receptor antagonist MK-801 or saline 20 min before the reactivation phase. MK-801 disrupted memory reconsolidation in standard (unchanged context) and novel (context change) reactivation conditions. Data are presented as average discrimination ratio \pm SEM. (*) $P<0.05$.

a fraction of a second, and more exploration actually occurred on MK-801 trials, in which rats were subsequently impaired.

\section{Experiment 3: Salient novel information is required during reactivation to destabilize memory traces following an extended acquisition period}

Experiment 3 assessed the effects on reconsolidation of enhancing the initial object-encoding phase. "Stronger" memories were induced by having rats explore the same sample objects over three separate sample phases on the same day. Twenty-four hours after the last sample phase, rats experienced one of the two reactivation conditions used in Experiment 2 (standard or novel), preceded by systemic injections of MK-801 or vehicle. This was followed $24 \mathrm{~h}$ later by the choice phase. Thus, beside the additional sample exploration, all aspects of Experiment 3 were identical to Experiment 2.

NMDA receptor blockade selectively disrupted reconsolidation in the novel reactivation condition (Fig. 3). The analysis indicated nonsignificant drug $(F<1)$ and reactivation condition $\left(F_{(1,19)}=3.74, \quad P=0.068\right)$ effects, but a significant interaction $\left(F_{(1,19)}=6.33, P<0.05\right)$. Post-hoc analyses indicated a significant difference between saline and MK-801 performance in the novel $\left(t_{(11)}=3.20, P<0.01\right)$ but not the standard $(t<1)$ reactivation condition. This result indicates that more strongly encoded object memory traces are resistant to the effects of NMDA receptor antagonism on reconsolidation unless they are reactivated in the presence of salient novel information.

Means ( \pm SEM) for the control measures taken from the sample, reactivation, and choice phases are presented in Tables $1-3$. The sample phase duration analysis indicated only a significant main effect of sample phase $\left(F_{(2,38)}=19.06, P<0.001\right)$, as rats took longer to reach the 30 -sec object exploration criterion in the second and third sample phases (Table 1). There was also a significant sample phase effect on total object exploration $\left(F_{(2,38)}=11.32, P<\right.$ $0.001)$, as rats generally explored less on the second and third samples (Table 1). As in Experiment 2, the reactivation phase duration analysis revealed a significant main effect of reactivation condition $\left(F_{(1,19)}=5.60\right.$, $P<0.05)$, as rats in the novel condition took longer to reach the 10 sec object exploration criterion (Table 2). There were, however, no significant differences in terms of total object exploration in the reactivation phase (all $F<1$; see Table 2 ). None of the other control measure analyses revealed any significant differences.

\section{Experiment 4: Salient novel information is required during reactivation to destabilize memory traces following a 48-h sample-to-reactivation interval}

Experiment 4 tested the effect of increasing the interval between the sample and reactivation phases. All procedures were identical to those in Experiment 2, except that there was a 48-h delay between the sample phase and the reactivation phase. Thus, compared with Experiment 2, the object memory was twice as old at the time of reactivation in Experiment 4.

Blockade of NMDA receptors selectively disrupted reconsolidation in the novel reactivation condition (Fig. 4). The ANOVA revealed a significant main effect of reactivation condition $\left(F_{(1,20)}=\right.$ 4.95, $P<0.05)$, but the drug $\left(F_{(1,20)}=3.24, P=0.087\right)$ and interaction $\left(F_{(1,20)}=2.99, P=0.099\right)$ terms did not reach significance. However, it is clear from Figure 4 that the significant effect is primarily accounted for by the impairment observed on MK-801 trials in the novel reactivation condition. Indeed, planned comparisons indicated a significant difference between saline and MK-801 performance in the novel $\left(t_{(10)}=2.53, P<0.05\right)$ but not the standard $(t<1)$ reactivation condition.

Means $( \pm$ SEM) for the control measures taken from the sample, reactivation, and choice phases are presented in Tables $1-3$. Of the control measures analyzed, only the main effect of reactivation condition in the reactivation phase duration analysis was significant $\left(F_{(1,20)}=4.64, P<0.05\right)$, as rats in the novel condition required more time to reach the 10 -sec object exploration criterion (Table 2). There were, however, no significant differences in terms of total object exploration in the reactivation phase (all $F<1$; see Table 2).

\section{Experiments 5 and 6: Extended (90-sec) "reactivation" period spent in experimental context in the absence of objects is not sufficient to destabilize older or stronger object memories}

Although there were no significant differences between the standard and novel reactivation conditions in terms of object exploration in the reactivation phases for Experiments 3 and 4, rats in

Table 2. Control measures collected from the reactivation phase for each drug condition and reactivation group from Experiments 1-4

\begin{tabular}{|c|c|c|c|c|c|}
\hline \multirow[b]{2}{*}{$\operatorname{Exp}$} & \multirow[b]{2}{*}{ Reactivation group } & \multicolumn{2}{|c|}{ Total object exploration } & \multicolumn{2}{|c|}{ Duration } \\
\hline & & Saline & MK-801 & Saline & MK-801 \\
\hline \multirow[t]{2}{*}{1} & No reactivation & - & - & - & - \\
\hline & Reactivation & $10 \pm 0$ & $9.7 \pm 0.3$ & $62.5 \pm 13.3$ & $80.1 \pm 14$ \\
\hline \multirow[t]{2}{*}{2} & Standard & $9.7 \pm 0.2$ & $10 \pm 0$ & $82.9 \pm 7$ & $69 \pm 7.7$ \\
\hline & Novel & $8.3 \pm 0.5$ & $9.4 \pm 0.4$ & $107.7 \pm 7.7$ & $95.6 \pm 7.6$ \\
\hline \multirow[t]{2}{*}{3} & Standard & $10 \pm 0$ & $9.3 \pm 0.7$ & $59.8 \pm 7$ & $75 \pm 9.1$ \\
\hline & Novel & $9.4 \pm 0.5$ & $9.5 \pm 0.4$ & $88 \pm 5.8$ & $86 \pm 8.3$ \\
\hline \multirow[t]{2}{*}{4} & Standard & $10 \pm 0$ & $9.6 \pm 0.4$ & $55.9 \pm 7.6$ & $50.7 \pm 8.6$ \\
\hline & Novel & $10 \pm 0$ & $9.9 \pm 0.1$ & $70.9 \pm 8$ & $73.5 \pm 6.8$ \\
\hline
\end{tabular}

Data are expressed as mean ( \pm SEM) of the total number of seconds spent exploring the objects and the total duration of the reactivation phase in seconds. In Experiments 5 and 6, the reactivation condition consisted of a 90 -sec reexposure to the empty Y-shaped apparatus; thus, no control measures were collected for these experiments (see Materials and Methods and Results sections for further details). 


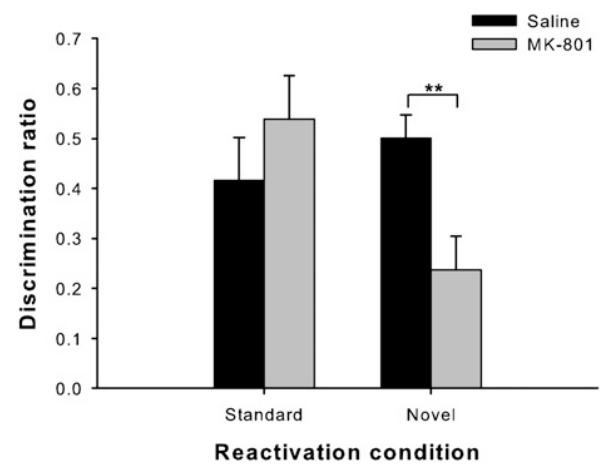

Figure 3. Strongly encoded object memories are destabilized upon reactivation $24 \mathrm{~h}$ after acquisition only when salient novel information is present during reactivation. Spontaneous object-recognition performance by rats in Experiment 3 on trials in which they received systemic injections of the NMDA receptor antagonist MK-801 or saline $20 \mathrm{~min}$ before the reactivation phase. MK-801 selectively disrupted memory reconsolidation in the novel (context change) reactivation condition. Data are presented as average discrimination ratio \pm SEM. $\left(^{* *}\right) P<0.01$.

the novel condition did require significantly more time to attain the 10-sec object exploration criterion in both experiments. It is possible that this extra time in the experimental context, and not the presence of novel information during reactivation, could explain the selective destabilization of object memories in the novel reactivation condition in Experiments 3 and 4 . Indeed, as noted above, Suzuki et al. (2004) have reported that otherwise resistant older or stronger fear-conditioned memories can be destabilized by extending the length of the reactivation phase in a contextual fear-conditioning paradigm. To rule out the extra time spent in the experimental context in the novel reactivation condition as an alternative explanation for the present results, we conducted two additional control experiments. In Experiment 5 , rats experienced an extended acquisition phase as in Experiment 3 to induce stronger object memories. In Experiment 6, there was a 48 -h delay between the sample phase and the reactivation phase as in Experiment 4 to induce older object memories. In both control experiments, the reactivation phase consisted of a 90-sec reexposure to the unchanged context (Y-shaped apparatus) from the sample phase. No objects were presented in the reactivation phase. A 90-sec period was chosen because this was the approximate length of the highest reactivation phase duration mean from Experiments 3 and 4 (87 sec in the novel reactivation condition from Experiment 3; see Table 2).

The results indicate that merely spending more time in the experimental context during the reactivation period is not sufficient to cause selective destabilization of older or stronger object memories (Fig. 5). The analysis of discrimination ratio data from Experiment 5 (Fig. 5A) indicated no significant difference in object recognition performance between saline and MK-801 trials $(t<1)$. Similarly, no significant effect of drug condition was observed in Experiment $6(t<1$; Fig. 5B). There were no significant effects in any of the control measures analyzed for either experiment, indicating that animals in both drug conditions demonstrated similar general object exploratory behavior in the sample and choice phases (see Tables 1 and 3). These findings further support the conclusion that the presence of novel contextual cues during memory reactivation increases the likelihood of older and stronger object memories to become destabilized.

\section{Discussion}

The present results synthesize and significantly extend recent findings concerning the nature of boundary conditions in mem- ory reconsolidation. Our results indicate that object recognition memory, like many other forms of memory, undergoes reconsolidation and that this process is subject to certain constraints related to the age or strength of the memory trace. The current findings, however, also emphasize the importance of the stimulus conditions prevailing during memory reactivation in relation to the influence of these putative boundary conditions. Although older and stronger memories may be less likely to become destabilized and vulnerable to disruption upon reactivation, in the present study the vulnerability of such memories was enhanced by changing the conditions under which memory reactivation occurred. One other recent report has shown that the presence of novel stimuli during reactivation can influence aspects of object memory reconsolidation; this study, however, limited its analysis to the role of the hippocampus in object memory reconsolidation (Rossato et al. 2007). The present results extend previous findings by demonstrating a clear interaction between the stimulus conditions during memory reactivation and the age or strength of the reactivated object memory.

Although additional sample object encoding may have occurred during the reactivation phase, the specificity of the druginduced deficit argues against an interpretation of the present results in terms of MK-801 blocking such encoding. If anything, additional sample object encoding should be more focused on the objects in the standard reactivation condition. If MK-801 injected before the reactivation phase significantly blocked additional object encoding, then we would expect to see object recognition impairments in rats administered MK-801 in the standard as well as the novel reactivation conditions. This was not the case. Thus, the pattern of the present findings suggests that MK-801 blocked reconsolidation of certain types of object memories following a specific reactivation protocol.

The present results are consistent with findings from other memory paradigms suggesting an important influence of memory age or strength on the reconsolidation process (Milekic and Alberini 2002; Eisenberg and Dudai 2004; Suzuki et al. 2004; Boccia et al. 2006; Frankland et al. 2006). For example, in a previous study, enhanced training or increased training-to-reactivation intervals reduced the likelihood of contextual fear-conditioned memories to undergo protein synthesis-dependent reconsolidation (Suzuki et al. 2004). Moreover, increasing the length of the reactivation phase reversed the effects of strengthening or aging the memory, rendering them vulnerable to destabilization (Suzuki

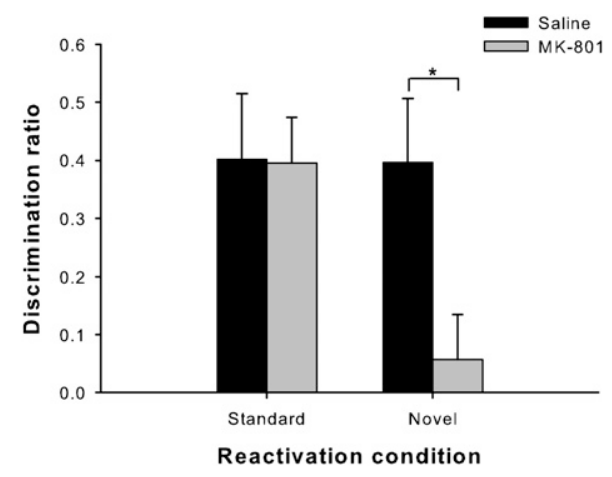

Figure 4. Older object memories are destabilized upon reactivation 48 $\mathrm{h}$ after acquisition only when salient novel information is present during reactivation. Spontaneous object-recognition performance by rats in Experiment 4 on trials in which they received systemic injections of the NMDA receptor antagonist MK-801 or saline 20 min before the reactivation phase. MK-801 selectively disrupted memory reconsolidation in the novel (context change) reactivation condition. Data are presented as average discrimination ratio \pm SEM. $\left(^{*}\right) P<0.05$. 
A

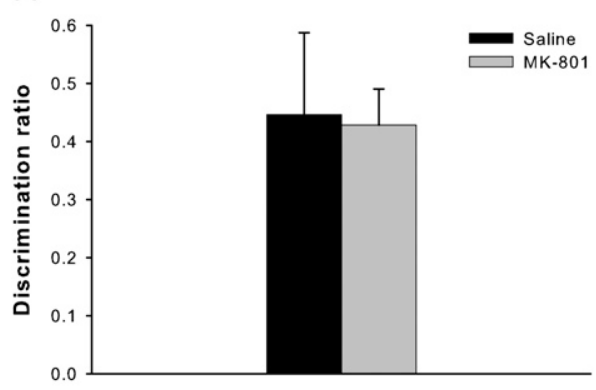

B

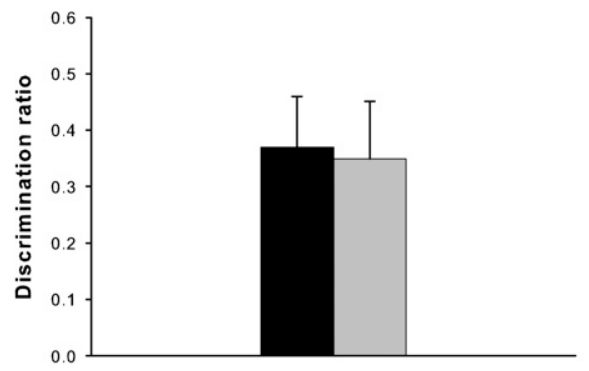

Figure 5. Older and stronger object memories are not selectively destabilized by a 90 -sec reexposure to the experimental context in the absence of objects. $(A)$ Choice phase object-recognition performance by rats in Experiment 5 on trials in which they received an extended acquisition period (three sample phases), followed $24 \mathrm{~h}$ later by a $90-$ $\mathrm{sec}$ reactivation period in the unchanged sample context ( $\mathrm{Y}$-shaped apparatus without floor insert) in the absence of any objects. (B) Choice phase object-recognition performance by rats in Experiment 6 on trials in which there was a 48-h interval between the single sample phase and the $90-\mathrm{sec}$ reactivation period, which was conducted in the same manner as in Experiment 5. In both experiments, systemic injections of the NMDA receptor antagonist MK-801 or saline were administered 20 min before the reactivation phase. MK-801 did not disrupt memory performance, indicating that the effects observed in Experiments 3 and 4 were not caused by the extra time spent in the Y-shaped apparatus by rats in the novel reactivation condition in those experiments. Data are presented as average discrimination ratio \pm SEM.

et al. 2004). Interestingly, we have demonstrated a similar result, not by changing the amount of reexposure to the encoded stimulus, but by modifying features of the physical context during reactivation. Older or more strongly encoded object memories reactivated in the presence of a salient novel contextual cue became destabilized and were disrupted by NMDA receptor blockade. Thus, although the present results support the suggestion that the vulnerability of a memory is inversely correlated with its age or strength, they also indicate that such a statement requires qualification with regard to the prevailing stimulus conditions under which the memory is reactivated. If explicit or salient novel information is present at the time of memory reactivation, certain aged or strong memories may nonetheless become vulnerable or modifiable.

The results of Experiments 1 and 2, as well as many previous studies (Nader et al. 2000a; Milekic and Alberini 2002; Eisenberg and Dudai 2004; Suzuki et al. 2004; Lee et al. 2006; Rossato et al. 2006; Lee and Everitt 2008), suggest that salient novel information is not always required during reactivation to destabilize a memory trace. These results, however, are not necessarily inconsistent with the suggestion that a dual encoding retrieval state is required to initiate the reconsolidation process (Morris et al. 2006). Perhaps the reactivation of a younger, weaker memory prompts additional encoding of the training stimulus. This additional, albeit less salient, novel encoding may be sufficient to destabilize relatively young or weak memory traces. This reasoning is consistent with the suggestion by Morris et al. (2006) that a novel encoding state is necessary to induce memory reconsolidation. Indeed, the present results support this assertion and extend it by demonstrating similar effects of novel encoding in a different type of memory task (object recognition compared with spatial memory) and by showing that the salience of novel information required during reactivation to produce memory destabilization may vary with the strength or age of the reactivated memory. Morris et al. (2006) showed that rats tested in a delayed matching-to-place task that required new memory encoding each day were vulnerable to anisomycin-induced blockade of memory reconsolidation, whereas rats trained to asymptotic levels of performance in a spatial reference memory task were not. Here, we report a similar result using a different method of inducing novel information encoding during memory reactivation and in a very different memory task, one that likely depends on unique brain circuitry. Thus, these two studies taken together imply an important general role for novel encoding processes in memory destabilization.

The present results therefore support the idea that one function of reconsolidation is to modify or update existing memories (Lewis 1979; Nader 2003; Dudai 2006; Morris et al. 2006). Our findings suggest that, at least for some kinds of memories, engagement of an information-encoding state is required for them to become destabilized. Indeed, it seems intuitive that better-trained (i.e., "stronger") memories might require the presence of more salient information during retrieval to become modifiable. Realworld memories for events or associations that are encountered more frequently than others are likely related to important regularities in an organism's environment. The ability to remember these regularities may have strong adaptive significance, and it could be disadvantageous to alter memories for such reliable associations unless significant behaviorally relevant new information is available. A substantial change to the context within which such associations have previously been encountered could be highly behaviorally relevant, and the present results suggest that this kind of explicit novel information can influence the likelihood of a relatively strong object memory to become destabilized. Once destabilized, such memories may be open to modification.

The question of why older memories are more resistant to destabilization has been addressed at length elsewhere (Dudai and Eisenberg 2004; Alberini 2005). It has been argued that such findings may indicate that the initial post-acquisition consolidation

Table 3. Total object (novel + familiar) exploration in the choice phase for each drug condition and reactivation group from Experiments 1-6

\begin{tabular}{llcc}
\hline & \multirow{2}{c}{$\begin{array}{c}c \\
\text { Exp }\end{array}$} & \multicolumn{2}{c}{ Total object exploration } \\
\cline { 3 - 4 } & group & Saline & MK-801 \\
\hline 1 & No reactivation & $11.1 \pm 1.7$ & $11.6 \pm 2.6$ \\
& Reactivation & $12.5 \pm 2.1$ & $8.8 \pm 2.2$ \\
2 & Standard & $12 \pm 1.3$ & $16.6 \pm 1.4$ \\
& Novel & $14.3 \pm 1.7$ & $13.4 \pm 1$ \\
3 & Standard & $16.2 \pm 1.6$ & $19.3 \pm 2.7$ \\
& Novel & $17.6 \pm 1.7$ & $19.9 \pm 1.9$ \\
4 & Standard & $14.1 \pm 1.6$ & $15.6 \pm 2.1$ \\
& Novel & $15.9 \pm 1.9$ & $16.2 \pm 2$ \\
5 & - & $16.9 \pm 2.2$ & $16.1 \pm 1.3$ \\
6 & - & $16.4 \pm 0.8$ & $15.2 \pm 1.3$ \\
& & &
\end{tabular}

Data are expressed as mean ( \pm SEM) of the total number of seconds spent exploring the objects. In Experiments 5 and 6, the reactivation condition consisted of a $90-\mathrm{sec}$ reexposure to the empty Y-shaped apparatus (see Materials and Methods and Results sections for further details). 
of memories may be a much more prolonged process than is commonly believed. The "lingering consolidation" hypothesis posits that this extended consolidation phase involves reorganization and updating of memories long after their original encoding (Dudai and Eisenberg 2004). According to this view, endogenous or retrieval-based reactivation of a memory promotes refinement and stabilization of the neural circuitry underlying the memory trace, but may also lead to the formation of "retrieval links" to other representations as the memory integrates with other information; reciprocal interactions between components of different neural circuits during this latter process may produce temporary instability (plasticity) in the existing circuitry (Dudai and Eisenberg 2004). Ultimately, this gradual, progressive stabilization of the memory may reach a point at which the memory can be considered stable, but the time course may be much longer than generally assumed, and the length of this period for specific memory types is likely linked to the acquisition protocol used (Dudai and Eisenberg 2004). Thus, the age-dependent gradient of "reconsolidation" impairment observed in the present study may reflect an effect of NMDA receptor blockade on a lingering consolidation process related to memory retrieval. The nature of the memory paradigm and the sampling protocol used may explain the relatively short duration of this gradient in the present study. Previous reports with rats and fish have demonstrated that a post-training period of $1 \mathrm{wk}$ or even much longer is required to observe aging-induced resistance to reconsolidation blockers (Milekic and Alberini 2002; Eisenberg and Dudai 2004; Suzuki et al. 2004; Boccia et al. 2006; Bustos et al. 2009). Clearly, different tasks produce memories with different temporal characteristics. Aversively motivated classical conditioning tasks such as fear conditioning and inhibitory avoidance, which account for most of the previous findings in this area, produce highly salient memories that persist for weeks or even months after training. Perhaps because the spontaneous object recognition task relies on exploratory behavior that is not explicitly reinforced, memory retention is generally not observed for more than a few days unless the sample exploration phase is substantially extended (Mumby et al. 2002, 2007; Gaskin et al. 2003). These critical task differences may explain the disparity in temporal gradients observed in the present and past studies. By $48 \mathrm{~h}$ after the sample phase, the object memories induced by the present protocol seem to have become relatively stabilized. Nonetheless, the introduction of a significant contextual change during reactivation produced a marked mismatch between the acquisition and reactivation environment, and this explicit novelty was sufficient to destabilize the memory circuitry.

This discussion highlights two critical implications of the present findings: First, despite the differences between tasks in terms of the specific temporal gradient, our results are consistent with many other studies in showing that memories become more resistant to destabilization as they age, and this may, therefore, be a general principle of memory storage and retrieval. Second, although older or more strongly encoded memories may become less vulnerable to destabilization, presentation of salient novel stimuli at the time of reactivation may render the memory once again susceptible to disruption or modification. A logical next step in this area of study is to address directly the question of whether salient novel information presented during memory reactivation is indeed incorporated into the original memory in a manner that alters subsequent behavior. Two recent studies have elegantly demonstrated such an effect in human episodic memory (Hupbach et al. 2007, 2008), but similar research in nonhuman animals remains scarce (Lukowiak et al. 2007; Lee 2008).

Several recent studies have emphasized the important point that memory destabilization and reconsolidation are two distinct but related processes (Ben Mamou et al. 2006; Lee et al. 2008;
Suzuki et al. 2008). Indeed, many reports now indicate that in order for the post-reactivation reconsolidation process to be initiated, the memory must first become destabilized under the appropriate reactivation conditions. The neural mechanisms underlying memory destabilization are clearly an important topic for future analysis, and recent reports have implicated several aspects of cellular and synaptic function in retrieval-mediated memory destabilization (Ben Mamou et al. 2006; Lee et al. 2008; Suzuki et al. 2008). These studies suggest that the mechanisms of memory destabilization are complex, and it is possible that the same mechanisms may not underlie destabilization of memories in all behavioral paradigms. The current findings may, in fact, speak to this latter suggestion. Here, we have shown that administration of an NMDA receptor antagonist 20 min prior to memory reactivation disrupts reconsolidation under certain conditions. This result seems at odds with a recent report that NMDA receptor antagonism can prevent the return of a consolidated fear memory to a labile state (Ben Mamou et al. 2006). This latter finding, however, was shown for a very different kind of memory (auditory fear conditioning), which depends critically on a different brain region (amygdala) from the behavior assessed in the current study. Object recognition memory is known to depend critically on the perirhinal cortex (Winters et al. 2008), and it is possible that NMDA receptor-mediated destabilization of consolidated memories is not a general mechanism of brain function. It also must be noted, however, that we cannot be absolutely certain of the time course of peak activation of MK-801 in the present study, and this may have occurred after the object trace was already destabilized. Further research will be required to assess the generality of the various mechanisms of memory destabilization, most of which have yet to be demonstrated in behavioral tasks other than fear conditioning.

Several final points are worth noting regarding the use of the spontaneous object recognition paradigm to study memory reconsolidation processes. First, unlike classical conditioning paradigms such as fear conditioning and inhibitory avoidance, which predominate in the field of memory reconsolidation, the object recognition task used here is not vulnerable to difficulties of interpretation related to extinction. In classical conditioning studies, memory retrieval can initiate two competing processes: reconsolidation of the original conditioned stimulus (CS)-unconditioned stimulus (US) association; or extinction, the formation of a new, inhibitory CS-no US association (Nader 2003; Dudai 2004; Suzuki et al. 2004). Several studies using classical conditioning paradigms have produced data that are inconsistent with a process of postretrieval memory reconsolidation (Berman and Dudai 2001; Lattal and Abel 2001; Vianna et al. 2001; Berman et al. 2003). In such studies, the memory reactivation parameters may have favored the extinction process rather than the reconsolidation process, in which case a putative amnesic agent such as anisomycin would be expected to block the establishment of the new CS-no US association rather than the reconsolidation of the original CSUS memory trace. Thus, the balance between extinction- and nonextinction-producing parameters is always an issue for reconsolidation studies using classical conditioning procedures (Eisenberg et al. 2003; Pedreira and Maldonado 2003). The object recognition paradigm used here measures familiarity-based changes in spontaneous exploratory behavior and, therefore, does not lend itself to the same criticism as classical conditioning tasks; there is no such thing as extinction per se for this type of object memory. A second advantage of the object recognition task is that, unlike fear conditioning and other paradigms commonly used in the reconsolidation literature, performance is not aversively motivated. It is important to consider a wide range of motivational factors and memory types when assessing the underlying principles of memory storage and retrieval. Finally, object recognition memory 
is considered by many to be a relatively pure test of declarative memory (Squire 1992; Manns et al. 2000, 2003), and the underlying neural circuitry differs from many of the other tasks used to study memory reconsolidation. Thus, analyses of object memory reconsolidation processes, side-by-side with the classical conditioning paradigms, will contribute to a more comprehensive understanding of the principles underlying memory reconsolidation.

The present study contributes important information about the nature of memory reconsolidation boundary conditions. Memory reactivation does not always automatically lead to complete destabilization. Whereas previous studies have demonstrated that merely lengthening the reactivation phase can destabilize otherwise resistant memories, the current results extend these findings by showing that such memories can be similarly destabilized by the presentation of novel contextual information at the time of memory reactivation. Further studies are required to assess the generality of the present findings, but at least in the case of object recognition memory there seems to be a critical interaction between the age or strength of a memory, its vulnerability to destabilization, and the conditions under which it is reactivated. The importance of salient novel information highlighted by the present study also supports the notion that memory reconsolidation is a process by which the brain can modify existing memories with new information.

\section{Materials and Methods}

\section{Subjects}

The subjects were 96 adult male Long-Evans rats (Charles River), weighing $\sim 395-415 \mathrm{~g}$ at the onset of each experiment. For each experiment, a group of experimentally naïve rats was used. Rats were housed in pairs and maintained on a reverse light/dark cycle (7:00 a.m. lights off; 7:00 p.m. lights on). All behavioral testing occurred during the dark phase of the cycle; however, the testing room was illuminated by ceiling-mounted fluorescent lights. During each experiment, rats received $20 \mathrm{~g}$ of rodent chow each evening to maintain $85 \%-90 \%$ of free-feeding body weight. Water was available ad libitum throughout the experiments. All procedures adhered to the guidelines of the Canadian Council on Animal Care and were approved by the Animal Care Committee at the University of Guelph.

\section{Drugs}

The NMDA receptor antagonist MK-801 ([+]-5-methyl-10,11-dihydro-SH-dibenzo[a,d]cyclohepten-5,10-imine maleate; Sigma) was dissolved in $0.9 \%$ physiological saline and administered by intraperitoneal (i.p.) injection at a concentration of $0.1 \mathrm{mg} / \mathrm{kg}$. Vehicle (0.9\% physiological saline) was administered in an identical manner. Drug injections were administered $\sim 20$ min before the reactivation phase in all experiments.

\section{Apparatus}

Spontaneous object recognition was conducted in a Y-shaped apparatus as described previously (Winters et al. 2004; Forwood et al. 2005). Briefly, the Y-shaped apparatus had high, homogenous white walls constructed from Plexiglas to prevent the rat from looking out into the room. The apparatus walls were $40-\mathrm{cm}$ high, while each arm was $27 \mathrm{~cm}$ in length and $10-\mathrm{cm}$ wide. The start arm contained a guillotine door $18 \mathrm{~cm}$ from the rear of the arm. This provided a start box area within which the rat could be confined at the start of a given trial. A video camera was mounted on a tripod above the apparatus to record all trials. Duplicate copies of objects made from plastic, ceramic, glass, and aluminum were obtained. The height of the objects ranged from 10 to $20 \mathrm{~cm}$, and they varied with respect to their visual and tactile qualities. All objects were affixed to the floor of the apparatus with an odorless reusable adhesive putty to prevent them from being displaced during testing. As far as could be determined, the objects had no natural significance for the rats, and they had never been associated with a reinforcer. Before being placed in the apparatus, objects were always wiped with 50\% ethanol.

\section{General procedure}

Each of the six experiments followed the same general procedure. Specific experimental manipulations are outlined below. All rats were habituated to the empty Y-shaped apparatus in two consecutive daily sessions. Each rat was individually brought to the experiment room in a transport cage and given a saline injection (i.p.). Immediately following the injection, the rat was taken to the Y-shaped apparatus, placed in the start box, and released into the main exploration area for $5 \mathrm{~min}$.

Behavioral testing began $24 \mathrm{~h}$ after the second habituation session. All rats were given two trials, with a minimum of $72 \mathrm{~h}$ between trials. Each trial consisted of three phases: sample, reactivation, and choice. Each phase occurred a minimum of $24 \mathrm{~h}$ apart, for a minimum retention delay (sample-to-choice) of $48 \mathrm{~h}$ per trial. A different object pair was used for each trial for a given rat, and the order of exposure to object pairs as well as the designated sample and novel objects for each pair were counterbalanced. Time spent exploring the objects was assessed using video recordings of the sample, reactivation, and choice phases. Data were collected by scoring exploratory bouts using a personal computer running a program written in Visual Basic 6.0.

In the sample phase, two identical objects (A1 and A2) were placed in the Y-shaped apparatus, one at the end of each exploration arm. Each rat was individually brought into the experiment room in a transport box and placed in the start box with the guillotine door lowered. The guillotine door was then raised to allow the rat into the exploration area of the maze. When the rat exited the start box, the guillotine door was lowered to prevent reentry, and the sample phase began. The time spent exploring the two objects was scored by an experimenter viewing the rat on a video screen. Exploration of an object was defined as directing the nose to the object at a distance of $<2 \mathrm{~cm}$ and/or touching it with the nose. The sample phase ended when the rat had explored the identical objects for a total of $30 \mathrm{sec}$, or after $3 \mathrm{~min}$ had passed, whichever came first. At the end of the sample phase, the rat was removed from the Y-shaped apparatus and was transported back to its home cage.

For the reactivation phase in each experiment, each rat was individually brought to the experiment room in a transport cage and in a separate area of the testing room behind a curtain, given an injection of either MK-801 or saline and then returned to its home cage. The experimenter was blind to the substance administered to each rat. Rats remained in their home cage for $20 \mathrm{~min}$, and were then brought back to the experiment room for behavioral testing (except for rats in the no-reactivation condition in Experiment 1, see below). For this phase, for Experiments 1-4, the Y-shaped apparatus contained the exact same objects shown to the rat during the sample phase: A1 in one arm and A2 in the other. The reactivation phase was carried out identically to the sample phase, except that it ended when the rat had explored the objects for a total of $10 \mathrm{sec}$, or when $2 \mathrm{~min}$ had passed, whichever came first. For Experiments 5 and 6, no objects were presented during the reactivation phase. Following the reactivation phase, the rat was removed and brought back to its home cage for $24 \mathrm{~h}$ until the choice phase.

In the choice phase, which occurred $24 \mathrm{~h}$ after the reactivation phase in all experiments, the Y-shaped apparatus contained a sample ("familiar") object (A1 or A2) in one arm and a new object (B) in the other. The exploration arms in which the choice objects were placed were counterbalanced between rats and across trials. The time spent exploring the novel and familiar objects was recorded for the 1-min choice phase. We then calculated a discrimination ratio, the proportion of total exploration time spent exploring the novel object (i.e., the difference in time spent exploring the novel and familiar objects divided by the total time spent exploring the objects), for each object recognition trial. This measure takes into account individual differences in the total amount of exploration time. Normal rats tended to explore the 
novel object more than the familiar sample object in this spontaneous object recognition paradigm.

\section{Experiment 1}

Experiment 1 was conducted to demonstrate the basic reconsolidation effect in the spontaneous object recognition task and to show that the predicted disruption of memory caused by MK-801 administration $24 \mathrm{~h}$ after the sample phase is reactivation dependent. Eleven rats were divided into two groups, a no-reactivation group $(n=5)$ and a reactivation group $(n=6)$. Animals in the reactivation group received MK-801 or saline injections $24 \mathrm{~h}$ after the sample phase and were placed back into the Y-shaped apparatus 20 min following the injection for a brief reactivation period, as described above. Rats in the no-reactivation condition received saline or MK-801 injections $24 \mathrm{~h}$ after the sample phase, but they did not experience the memory reactivation session. This no-reactivation condition controls for the possibility of lingering consolidation effects $24 \mathrm{~h}$ after memory acquisition. All rats were run on two separate trials, one in which MK-801 was administered and one in which they received saline, and the order of MK-801 and saline trials was counterbalanced. The sample-to-choice retention interval was $48 \mathrm{~h}$.

\section{Experiment 2}

The purpose of Experiment 2 was to determine the effect on reconsolidation of reactivating the sample object memory trace in the presence or absence of novel contextual information. Thus, two reactivation conditions were analyzed, each occurring $24 \mathrm{~h}$ after the sample phase. In the standard reactivation condition, the sample objects were presented in the Y-shaped apparatus as described above; the apparatus was unchanged from the sample phase. In the novel reactivation condition, the sample objects were represented to the rats in the same Y-shaped apparatus, but with one significant contextual modification. An insert constructed of white foam board and covered in no-slip rubber padding was placed inside the apparatus. The insert was $10-\mathrm{cm}$ wide, and extended $38 \mathrm{~cm}$ from inside the start box into the exploration area. The floor insert did not extend into the arms containing the objects, but covered the entire "middle stem" of the Y-shaped apparatus, extending beyond the start box into the exploratory area. Two separate groups of rats were run for Experiment 2, one group in the standard reactivation condition $(n=12)$ and one group in the novel reactivation condition $(n=10)$. All rats were run on two separate trials, one in which MK-801 was administered and one in which they received saline. All injections were conducted $\sim 20 \mathrm{~min}$ before the start of the reactivation phase, and the order of MK-801 and saline trials was counterbalanced. The sample-to-choice retention interval was $48 \mathrm{~h}$.

\section{Experiment 3}

Experiment 3 assessed the effects of enhancing the initial objectencoding phase. More strongly encoded memories were induced by having rats explore the same sample objects over three separate sample phases on the same day. The interval between sample phases was approximately $1 \mathrm{~h}$. Each sample phase was run identically, as described above. Twenty-four hours after the last sample phase, rats experienced one of the two reactivation conditions used in Experiment 2 (standard, $n=9$; novel, $n=12$ ). Each reactivation phase was preceded 20 min earlier by injection of either MK-801 or saline. All rats were run on two trials, one with each drug condition, in counterbalanced fashion. Aside from the additional sample exploration, all aspects of Experiment 3 were identical to Experiment 2. The sample-to-choice retention interval was $48 \mathrm{~h}$.

\section{Experiment 4}

Experiment 4 tested the effect of increasing the interval between the sample and reactivation phases. All procedures were identical to those in Experiment 2, except that there was a 48-h delay between the sample acquisition phase and the reactivation phase. Thus, compared with Experiment 2, the object memory was twice as old at the time of reactivation in Experiment 4. There were 11 rats in each reactivation condition (standard, novel). The sampleto-choice retention interval was $72 \mathrm{~h}$.

\section{Experiments 5 and 6}

Experiments 5 and 6 were conducted as control experiments to address the possibility that the unintended extended duration of the reactivation phase observed for rats in the novel reactivation condition in Experiments 3 and 4 could explain the selective destabilization of memories in this condition rather than the presence of novel contextual information. Experiments 5 and 6 were run identically to Experiments 3 and 4, respectively, except for one significant modification. To assess whether merely spending more time in the testing context would be sufficient to destabilize older or stronger memories, the reactivation phase was conducted as a 90-sec reexposure to the unchanged sample context (the Y-shaped apparatus without the floor insert) 24 (Experiment 5) or $48 \mathrm{~h}$ (Experiment 6) after the sample phase. A 90-sec period was chosen because this was the approximate length of the highest reactivation phase duration mean from Experiments 3 and 4 (87 sec in the novel reactivation condition from Experiment 3; see Table 2). No objects were present in the Y-shaped apparatus during the reactivation phase in Experiments 5 and 6. Ten rats were run in each experiment, and each rat was run for two trials, one trial with a saline injection and one trial with an MK-801 injection, each $20 \mathrm{~min}$ before the reactivation phase.

\section{Data analysis}

Group means of six measures taken from object recognition testing were analyzed for Experiments 1-4. Two measures were analyzed from each phase of behavioral testing: the total object exploration in the sample phase and the duration of the sample phase; the total object exploration and duration of the reactivation phase; the total object exploration and the discrimination ratio from the choice phase. The total exploration and duration control measures were assessed to ensure the absence of any preexisting or drug-induced differences in general exploratory behavior in the sample, reactivation, or choice phases, differences that could influence recognition memory performance. Means from these measures were submitted to two-way (drug $\times$ reactivation condition) mixed-factorial ANOVAs, except in Experiment 1, where the reactivation exploration analyses were conducted as paired-samples $t$-tests on the reactivation group data. For Experiment 3, the duration and total object exploration for all three sample phases were assessed. The sample analyses for Experiment 3 were conducted as three-way (drug $\times$ reactivation condition $\times$ sample phase) mixed-factorial ANOVAs. Paired-samples $t$-tests were used for post-hoc analyses and planned comparisons. Experiments 5 and 6 did not involve a typical reactivation phase with object presentation, so no control measures were gathered from this phase. The total object exploration and the discrimination ratio from the choice phase for each of Experiments 5 and 6 were analyzed by paired-samples $t$-tests. Total object exploration in the sample phase and the duration of the sample phase were each also analyzed with paired-samples $t$-tests for Experiment 5; for Experiment 6 , these sample phase control measures were each analyzed using two-way (drug $\times$ sample phase) ANOVAs with repeated measures. All statistical analyses were conducted with a significance level of $\alpha=0.05$.

\section{Acknowledgments}

This research was supported by a National Sciences and Engineering Research Council (NSERC) of Canada Discovery Grant to B.D.W. and an NSERC Undergraduate Student Research Award to M.C.T. We thank James Reid for running Experiments 5 and 6.

\section{References}

Abe H, Ishida Y, Iwasaki T. 2004. Perirhinal N-methyl-D-aspartate and muscarinic systems participate in object recognition in rats. Neurosci Lett 356: 191-194. 
Alberini CM. 2005. Mechanisms of memory stabilization: Are consolidation and reconsolidation similar or distinct processes? Trends Neurosci 28: 51-56.

Anokhin KV, Tiunova AA, Rose SP. 2002. Reminder effects-reconsolidation or retrieval deficit? Pharmacological dissection with protein synthesis inhibitors following reminder for a passive-avoidance task in young chicks. Eur I Neurosci 15: 1759-1765.

Barker GR, Warburton EC, Koder T, Dolman NP, More JC, Aggleton JP, Bashir ZI, Auberson YP, Jane DE, Brown MW. 2006. The different effects on recognition memory of perirhinal kainate and NMDA glutamate receptor antagonism: Implications for underlying plasticity mechanisms. J Neurosci 26: 3561-3566.

Ben Mamou C, Gamache K, Nader K. 2006. NMDA receptors are critical for unleashing consolidated auditory fear memories. Nat Neurosci 9: 1237-1239.

Berman DE, Dudai Y. 2001. Memory extinction, learning anew, and learning the new: Dissociations in the molecular machinery of learning in cortex. Science 291: 2417-2419.

Berman DE, Hazvi S, Stehberg J, Bahar A, Dudai Y. 2003. Conflicting processes in the extinction of conditioned taste aversion: Behavioral and molecular aspects of latency, apparent stagnation, and spontaneous recovery. Learn Mem 10: 16-25.

Boccia MM, Blake MG, Acosta GB, Baratti CM. 2006. Post-retrieval effects of icv infusions of hemicholinium in mice are dependent on the age of the original memory. Learn Mem 13: 376-381.

Brown TE, Lee BR, Sorg BA. 2008. The NMDA antagonist MK-801 disrupts reconsolidation of a cocaine-associated memory for conditioned place preference but not for self-administration in rats. Learn Mem 15: 857-865.

Bustos SG, Maldonado H, Molina VA. 2009. Disruptive effect of midazolam on fear memory reconsolidation: Decisive influence of reactivation time span and memory age. Neuropsychopharmacology 34: 446-457.

Dudai Y. 2004. The neurobiology of consolidations, or, how stable is the engram? Annu Rev Psychol 55: 51-86.

Dudai Y. 2006. Reconsolidation: The advantage of being refocused. Curr Opin Neurobiol 16: 174-178.

Dudai Y, Eisenberg M. 2004. Rites of passage of the engram: Reconsolidation and the lingering consolidation hypothesis. Neuron 44: 93-100.

Eisenberg M, Dudai Y. 2004. Reconsolidation of fresh, remote, and extinguished fear memory in medaka: Old fears don't die. Eur J Neurosci 20: 3397-3403.

Eisenberg M, Kobilo T, Berman DE, Dudai Y. 2003. Stability of retrieved memory: Inverse correlation with trace dominance. Science 301: 1102-1104.

Forwood SE, Winters BD, Bussey TJ. 2005. Hippocampal lesions that abolish spatial maze performance spare object recognition memory at delays of up to 48 hours. Hippocampus 15: 347-355.

Frankland PW, Ding HK, Takahashi E, Suzuki A, Kida S, Silva AJ. 2006. Stability of recent and remote contextual fear memory. Learn Mem 13: $451-457$.

Gaskin S, Tremblay A, Mumby DG. 2003. Retrograde and anterograde object recognition in rats with hippocampal lesions. Hippocampus 13: 962-969.

Hupbach A, Gomez R, Hardt O, Nadel L. 2007. Reconsolidation of episodic memories: A subtle reminder triggers integration of new information. Learn Mem 14: 47-53.

Hupbach A, Hardt O, Gomez R, Nadel L. 2008. The dynamics of memory: Context-dependent updating. Learn Mem 15: 574-579.

Lattal KM, Abel T. 2001. Different requirements for protein synthesis in acquisition and extinction of spatial preferences and context-evoked fear. J Neurosci 21: 5773-5780.

Lee JL. 2008. Memory reconsolidation mediates the strengthening of memories by additional learning. Nat Neurosci 11: 1264-1266.

Lee JL, Everitt BJ. 2008. Appetitive memory reconsolidation depends upon NMDA receptor-mediated neurotransmission. Neurobiol Learn Mem 90: 147-154.

Lee JL, Everitt BJ, Thomas KL. 2004. Independent cellular processes for hippocampal memory consolidation and reconsolidation. Science $\mathbf{3 0 4}$ 839-843.

Lee JL, Milton AL, Everitt BJ. 2006. Reconsolidation and extinction of conditioned fear: Inhibition and potentiation. J Neurosci 26: 10051-10056.

Lee SH, Choi JH, Lee N, Lee HR, Kim JI, Yu NK, Choi SL, Kim H, Kaang BK. 2008. Synaptic protein degradation underlies destabilization of retrieved fear memory. Science 319: 1253-1256.

Lewis DJ. 1979. Psychobiology of active and inactive memory. Psychol Bull 86: $1054-1083$.

Lukowiak K, Fras M, Smyth K, Wong C, Hittel K. 2007. Reconsolidation and memory infidelity in Lymnaea. Neurobiol Learn Mem 87: 547-560.

Manns JR, Stark CE, Squire LR. 2000. The visual paired-comparison task as a measure of declarative memory. Proc Natl Acad Sci 97: 12375-12379.

Manns JR, Hopkins RO, Reed JM, Kitchener EG, Squire LR. 2003. Recognition memory and the human hippocampus. Neuron 37: 171-180.
McGaugh JL. 1966. Time-dependent processes in memory storage. Science 153: $1351-1358$.

McGaugh JL. 2000. Memory-a century of consolidation. Science 287: 248-251.

Milekic MH, Alberini CM. 2002. Temporally graded requirement for protein synthesis following memory reactivation. Neuron 36: 521-525.

Misanin JR, Miller RR, Lewis DJ. 1968. Retrograde amnesia produced by electroconvulsive shock after reactivation of a consolidated memory trace. Science 160: 554-555.

Morris RG, Inglis J, Ainge JA, Olverman HJ, Tulloch J, Dudai Y, Kelly PA. 2006. Memory reconsolidation: Sensitivity of spatial memory to inhibition of protein synthesis in dorsal hippocampus during encoding and retrieval. Neuron 50: 479-489.

Mumby DG, Glenn MJ, Nesbitt C, Kyriazis DA. 2002. Dissociation in retrograde memory for object discriminations and object recognition in rats with perirhinal cortex damage. Behav Brain Res 132: 215-226.

Mumby DG, Piterkin P, Lecluse V, Lehmann H. 2007. Perirhinal cortex damage and anterograde object recognition in rats after long retention intervals. Behav Brain Res 185: 82-87.

Nader K. 2003. Memory traces unbound. Trends Neurosci 26: 65-72.

Nader K, Schafe GE, Le Doux JE. 2000a. Fear memories require protein synthesis in the amygdala for reconsolidation after retrieval. Nature 406: 722-726.

Nader K, Schafe GE, LeDoux JE. 2000b. The labile nature of consolidation theory. Nat Rev Neurosci 1: 216-219.

Pedreira ME, Maldonado H. 2003. Protein synthesis subserves reconsolidation or extinction depending on reminder duration. Neuron 38: $863-869$.

Pedreira ME, Perez-Cuesta LM, Maldonado H. 2002. Reactivation and reconsolidation of long-term memory in the crab Chasmagnathus: Protein synthesis requirement and mediation by NMDA-type glutamatergic receptors. J Neurosci 22: 8305-8311.

Przybyslawski J, Sara SJ. 1997. Reconsolidation of memory after its reactivation. Behav Brain Res 84: 241-246.

Rodriguez-Ortiz CJ, De la Cruz V, Gutiérrez R, Bermudez-Rattoni F. 2005. Protein synthesis underlies post-retrieval memory consolidation to a restricted degree only when updated information is obtained. Learn Mem 12: 533-537.

Rodriguez-Ortiz CJ, Garcia-DeLaTorre P, Benavidez E, Ballesteros MA, Bermudez-Rattoni F. 2008. Intrahippocampal anisomycin infusions disrupt previously consolidated spatial memory only when memory is updated. Neurobiol Learn Mem 89: 352-359.

Rossato JI, Bevilaqua LR, Medina JH, Izquierdo I, Cammarota M. 2006. Retrieval induces hippocampal-dependent reconsolidation of spatial memory. Learn Mem 13: 431-440.

Rossato JI, Bevilaqua LR, Myskiw JC, Medina JH, Izquierdo I, Cammarota M. 2007. On the role of hippocampal protein synthesis in the consolidation and reconsolidation of object recognition memory. Learn Mem 14: 36-46.

Sara SJ. 2000. Retrieval and reconsolidation: Toward a neurobiology of remembering. Learn Mem 7: 73-84.

Squire LR. 1992. Memory and the hippocampus: A synthesis from findings with rats, monkeys, and humans. Psychol Rev 99: 195-231.

Suzuki A, Josselyn SA, Frankland PW, Masushige S, Silva AJ, Kida S. 2004. Memory reconsolidation and extinction have distinct temporal and biochemical signatures. J Neurosci 24: 4787-4795.

Suzuki A, Mukawa T, Tsukagoshi A, Frankland PW, Kida S. 2008. Activation of LVGCCs and CB1 receptors required for destabilization of reactivated contextual fear memories. Learn Mem 15: 426-433.

Torras-Garcia M, Lelong J, Tronel S, Sara SJ. 2005. Reconsolidation after remembering an odor-reward association requires NMDA receptors. Learn Mem 12: 18-22.

Vianna MR, Szapiro G, McGaugh JL, Medina JH, Izquierdo I. 2001. Retrieval of memory for fear-motivated training initiates extinction requiring protein synthesis in the rat hippocampus. Proc Natl Acad Sci 98: 12251-12254.

von Hertzen LS, Giese KP. 2005. Memory reconsolidation engages only a subset of immediate-early genes induced during consolidation. J Neurosci 25: 1935-1942.

Winters BD, Bussey TJ. 2005. Glutamate receptors in perirhinal cortex mediate encoding, retrieval, and consolidation of object recognition memory. J Neurosci 25: 4243-4251.

Winters BD, Forwood SE, Cowell RA, Saksida LM, Bussey TJ. 2004. Doubledissociation between the effects of peri-postrhinal cortex and hippocampal lesions on tests of object recognition and spatial memory: Heterogeneity of function within the temporal lobe. J Neurosci 24: 5901-5908.

Winters BD, Saksida LM, Bussey TJ. 2008. Object recognition memory: Neurobiological mechanisms of encoding, consolidation and retrieval. Neurosci Biobehav Rev 32: 1055-1070.

Received June 12, 2009; accepted in revised form June 22, 2009. 


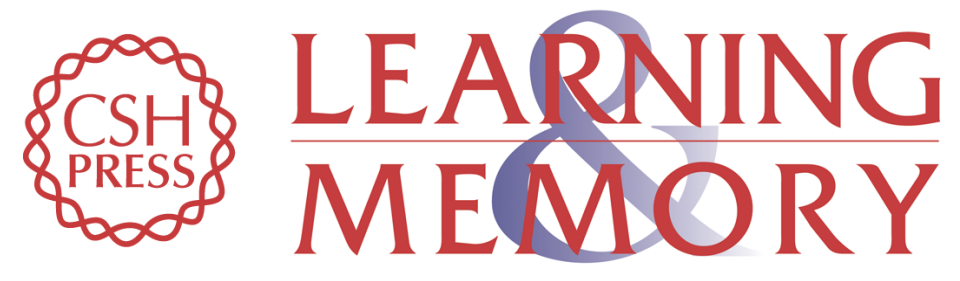

\section{Older and stronger object memories are selectively destabilized by reactivation in the presence of new information}

Boyer D. Winters, Mark C. Tucci and Melynda DaCosta-Furtado

Learn. Mem. 2009, 16:

Access the most recent version at doi:10.1101//m.1509909

References This article cites 57 articles, 29 of which can be accessed free at:

http://learnmem.cshlp.org/content/16/9/545.full.html\#ref-list-1

License

Email Alerting Receive free email alerts when new articles cite this article - sign up in the box at the Service top right corner of the article or click here. 